\title{
INVESTIGATION OF BLOOD FLOW THROUGH AN ARTERY IN THE PRESENCE OF OVERLAPPING STENOSIS
}

\section{K. Maruthi Prasad ${ }^{1}$, T. Sudha ${ }^{2 *}$, M. V. Phanikumari ${ }^{3}$}

1, 2,3 Department of Mathematics, School of Technology, GITAM University, Hyderabad (T.S), India-502329.

Email: Maruthiprad.karanamu@gitam.edu, sudhathulluri@gmail.com, phanin_mouli@yahoo.co.in

\begin{abstract}
:
The effects of an overlapping stenosis on blood flow characteristics in an artery have been studied. Blood has been represented by a couple stress fluid. The flow equations have been linearized and the expressions for pressure drop, resistance to the flow and wall shear stress have been derived. The results are shown graphically. It is observed that the resistance to the flow, pressure drop and wall shear stress increases with height and length of the stenosis. And it is noticed that the resistance to the flow and pressure drop decreases with couple stress fluid parameters. But wall shear stress increases with couple stress fluid parameters.
\end{abstract}

Keywords: Resistance to the flow, stenosis, couple stress fluid, couple stress fluid parameters.

\section{NOMENCLATURE}

$I_{0}, I_{1} \quad$ Modified Bessel functions of order zero and one

$L \quad$ Length of the tube

$L_{0} \quad$ Length of the stenosis

$p \quad$ Pressure

$q \quad$ Flow flux

$R(z) \quad$ Radius of the tube with stenosis

$R_{0}(z) \quad$ Radius of the tube without stenosis

$u \quad$ Axial velocity

\section{Greek symbols}

$\delta \quad$ Maximum height of the stenosis

$\bar{\alpha}, \bar{\eta} \quad$ Couple stress fluid parameters

$\mu \quad$ Viscosity of the couple stress fluid

$\Delta p \quad$ Pressure drop

$\lambda$ Resistance to the flow

$\bar{\lambda} \quad$ Normalized resistance to the flow

$\tau_{w} \quad$ Wall shear stress

$\bar{\tau}_{w} \quad$ Normalized wall shear stress

\section{Introduction}

In the present world one of the major health problems is atherosclerosis or stenosis. It is well known that blood flow through a stenosed artery can cause an obstruction in the blood flow which causes serious circulatory disorders. In this condition flow behavior is quite different from that in a normal artery. The main reason for this situation is formation of plaques, fatty substances and cholesterol in the blood vessels is known as atherosclerosis or stenosis.

Stenosis can cause severe circulatory disorders by preventing the blood supply. For example stenotic artery supplying blood to brain can bring about cerebral strokes. Similarly in the coronary artery which supplies blood to the heart when stenosis is formed in these arteries it can cause myocardial infarction leading to the results heart failure. Once obstruction has developed it results into significant changes in pressure distribution, wall shear stress and flow of resistance, with this knowledge it is believed that hydrodynamic factors like impedance, wall shear stress etc play a pivotal role in the development and progression of arterial stenosis.

To understand the effects of stenosis in artery many researchers (Texon, 1957, Young, 1968), Lee and Fung, 1970, Fry, 1972, Zendehbudi and Moayeri, 1999 and Radhakrishnamacharya et al., 2007) have analyzed mathematical models for blood flow through arteries under diseased conditions by assuming blood as Newtonian fluid. Blood behaves as Newtonian fluid when it flows through larger diameter blood vessels at high shear rate, but it is well recognized that blood behaves like a non-Newtonian fluid under certain flow conditions, 1813-8235 (Print), 2070-8998 (Online) @ 2017 ANAME Publication. All rights reserved. $\quad$ Received on: January, 2017 
particularly at low shear rates and in smaller diameter blood vessels. In the view of this several authors (Chaturani and Swamy, 1986, Sapna, 2009 and Verma, 2014) are studied the blood flow characteristics for stenosis by considering blood as non-Newtonian fluid. And all these studies considered the effect of single stenosis. A survey of the literature on stenotic development indicates that most of the studies in the literature are mainly concerned with single symmetric or non-symmetric stenoses. In a realistic situation, however, the constrictions may develop in series (multiple stenoses), may be of irregular shapes, overlapping, composite in nature, etc.

Based on this Chakravarthy and Mandal (1994) presented the mathematical modeling of blood flow through an overlapping arterial stenosis. The non-Newtonian arterial blood flow through an overlapping stenosis was investigated by Srivastava et al. (2010). Nanda et al. (2012) studied a non - Newtonian two-phase fluid model for blood flow through arteries under stenotic condition. Maruthiprasad et al. (2014) analyzed a mathematical model of over lopping stenosis by considering blood flow as Herschel-Bulkley fluid. A casson flow of blood through an arterial tube with overlapping stenosis presented by Arunkumar (2015).

Couple stress fluid theory proposed by Stokes (1966). Couple stress fluid is a special case of the non-Newtonian fluids and this fluid represents the simplest generalization of the classical viscous fluid theory that sustains couple stresses and the body couples. The important feature of these fluids is that stress tensor is not symmetric and their accurate flow behavior cannot be predicted by classical Newtonian theory and the main effect of couple stress will introduce a size dependent effect that is not present in the classical viscous theories. Studies on the couple stress fluid behavior are very useful to describe human/sub- human blood. The couple stress model is widely used because of its mathematical simplicity compared with other models.

Several researchers studied on couple stress fluid flows under different conditions. Taking few on this, Sahu et al. (2010) presented a study of arterial blood flow in stenosed vessel using couple stress fluid. Srinivasacharya and Srikanth (2012) investigated steady streaming effect on the flow of a couple stress fluids through a constricted annulus. The effect of slip condition on couple stress fluid flow through porous medium with stenosis analyzed by Gurju and Radhakrishnamacharya (2013). Raju et al., (2014) studied Soret effects due to natural convection in a non-Newtonian fluid flow in porous medium with heat and mass transfer. The effect of the couple stress fluid flow on MHD peristaltic motion with uniform porous medium in the presence of slip effect was investigated by Kumar (2015). Hamza et al. (2016) studied on steady flow of couple stress fluid in constricted tapered artery, effects of transverse magnetic field moving catheter and slip velocity. Srinivasacharya and Madhavarao (2016) investigated on Pulsatile flow of couple stress fluid through a bifurcated artery.

Keeping in this view, a mathematical model for blood flow through a narrow tube in the presence of overlapping stenosis has been investigated. Here blood is treated as a couple stress fluid. The arterial wall segment is considered to be rigid as well as deformable. The wall in the vicinity of the stenosis is usually relatively rigid when stenosis develops in human vasculature. The arterial length is considered to large enough as compared to the radius, so that the entrance, end and special wall effects can be neglected.

\section{Mathematical Formulation}

Consider the steady flow of blood through an axially symmetric but radially non symmetric overlapping stenosed artery. The geometry of Stenosis can be taken as (Srivastava, 2011)

$$
\begin{aligned}
h=\frac{R(z)}{R_{0}} & =1-\frac{3 \delta}{2 R_{0} L_{0}^{4}}\left[11(z-d) L_{0}^{3}-47(z-d)^{2} L_{0}^{2}+72(z-d)^{3} L_{0}-36(z-d)^{4}\right], d \leq z \leq d+L_{0} \\
& =1 \text {, otherwise. }
\end{aligned}
$$

Where $R(z)$ the radius of the tube with stenosis, $R_{0}(z)$ is the radius of the tube without stenosis, $L_{0}$ is the length of the stenosis and $d$ indicates the stenosis location, $\delta$ is the maximum height of the stenosis. Projection of stenosis at the two positions is denoted by $z$ as $z=d+\frac{L_{0}}{6}, z=d+\frac{5 L_{0}}{6}$ respectively. The critical height is taken as $\frac{3 \delta}{4}$ at $z=d+L_{0} / 2$, from the origin.

Investigation of blood flow through an artery in the presence of overlapping stenosis 


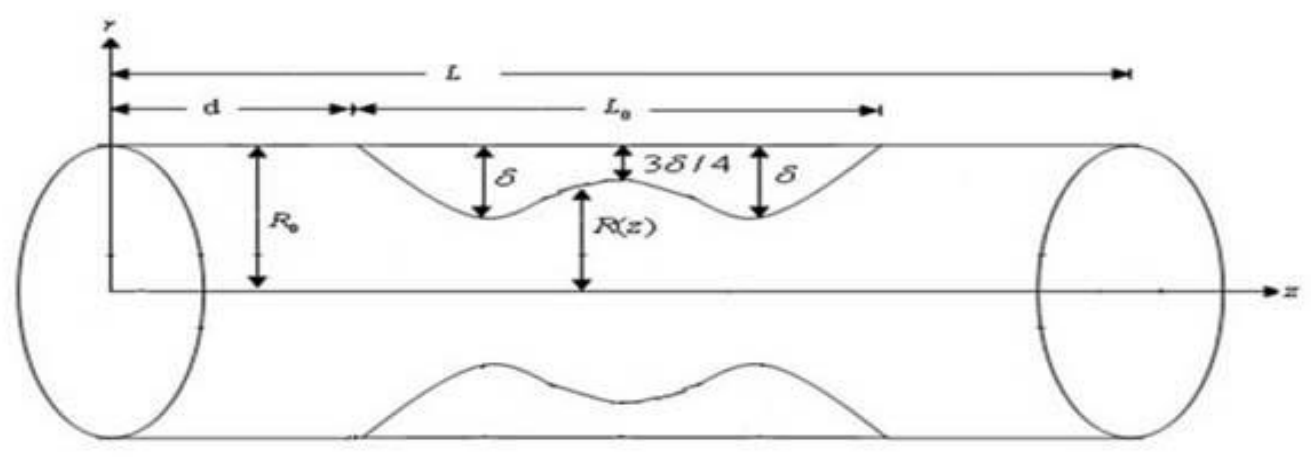

Fig. 1: Geometry of a uniform tube of circular cross section with overlapping stenosis

The constitutive equations and equations of motion for couple stress fluid flow are (Stokes, 1966) $\operatorname{div} \bar{q}=0$

$T_{j i, j}=\rho \frac{d u_{i}}{d t}, \quad e_{i j k} T_{j k}^{A}+M_{j i, j}=0$

$l_{i j}=-p \delta_{i j}+2 \mu_{i j} d_{i j}, \mu_{i j}=4 \eta u_{j, i}+4 \eta^{\prime} u_{i j}$

Where $u_{i}$ is the velocity vector; $T_{i j}$ and $T_{i j}^{A}$ are the symmetric and antisymmetric parts of stress tensor $T_{i j}$ respectively; $M_{i j}$ is the couple stress tensor; $\mu_{i j}$ is the deviatoric part of $M_{i j}$; and $u_{i}$ is the vorticity vector; $d_{i j}$ is the symmetric part of the velocity gradient; $\eta$ and $\eta^{\prime}$ are constants associated with the couple stress; $p$ is the pressure and other terms have their usual meaning from tensor analysis. Assume that the stenosis is to be mild. The following restrictions are to be satisfied $\delta_{i} \ll \min \left(R_{0}, R(z)\right), \delta_{i} \ll L_{i}$ and apply the following nondimensional quantities in Eq. (2) and Eq. (3)

$\bar{r}=\frac{r}{a}, \quad \bar{z}=\frac{z}{L}, \bar{\delta}=\frac{\delta}{R_{0}}, \quad \bar{p}=\frac{a^{2} p}{L \mu U}$

$\bar{\alpha}=a \alpha=a \sqrt{\frac{\mu}{\eta}}, \bar{U}=\frac{u}{U^{\prime}}$

$\bar{d}=\frac{d}{L}, \bar{L}=\frac{L}{L_{0}}$

Where $U$ represents the average velocity.

Then Eq. (2) and Eq. (3) become

$\frac{\partial u}{\partial r}+\frac{u}{r}+\frac{\partial u}{\partial z}=0$

$\frac{\partial p}{\partial r}=0$

$\frac{1}{r} \frac{\partial}{\partial r}\left\{r \frac{\partial}{\partial r}\left(1-\frac{1}{\bar{\alpha}^{2}} \nabla^{2}\right) u\right\}=\frac{\partial p}{\partial z}$

Where $\nabla^{2}=\frac{1}{r} \frac{\partial}{\partial r}\left(r \frac{\partial}{\partial r}\right)$, and $u$ is the velocity

$\bar{\alpha}^{2}=\frac{\mu}{\eta} a^{2}$ (Couple stress parameter), since $\sqrt{\frac{\mu}{\eta}}$, a characteristic measure of polarity of the fluid model, has a dimension of length, $\bar{\alpha}$ indicates the ratio of the tube to material characteristic length $\sqrt{\frac{\eta}{\mu}}$, in the limit as $\eta \rightarrow 0$, i.e, $\bar{\alpha} \rightarrow \infty$, Eq. (7) reduces to classical Navier-Stokes equation.

The non-dimensional boundary conditions are

$\frac{\partial u}{\partial r}=0$ at $r=0$

$\frac{\partial^{2} u}{\partial r^{2}}-\frac{\eta}{r} \frac{\partial u}{\partial r}=0$ at $r=h(z)$

$\frac{\partial^{2} u}{\partial r^{2}}-\frac{\eta}{r} \frac{\partial u}{\partial r}=$ is finite at $r=0$

That implies $u$ is finite at $r=0$

$u=0$ at $r=h(z)$

Investigation of blood flow through an artery in the presence of overlapping stenosis 


\section{Solution}

Solving Eq. (5) and Eq. (7) under the boundary conditions (8) to (12), we obtain the velocity as

$u=A I_{0}(\bar{\alpha} r)+\left(\frac{r^{2}}{4}+\frac{1}{\bar{\alpha}^{2}}\right) \frac{d p}{d z}-d \bar{\alpha}^{2}$

Where $A=\frac{h\left(\frac{\eta-1}{2}\right) \frac{d p}{d z}}{h \bar{\alpha}^{2} I_{0}(\bar{\alpha} h)-\bar{\alpha} I_{1}(\bar{\alpha} h)-\eta \bar{\alpha} I_{1}(\bar{\alpha} h)}$

$d=\frac{1}{\bar{\alpha}^{2}}\left[A I_{0}(\bar{\alpha} h)+\left(\frac{h^{2}}{4}+\frac{1}{\bar{\alpha}^{2}}\right) \frac{d p}{d z}\right]$

Where $I_{0}$ and $I_{1}$ are Modified Bessel functions of order zero and one respectively.

The dimensionless flux $q=\frac{q^{\prime}}{\pi a^{2} c}$ is given by

$q=\int_{0}^{h} 2 r u d r$

Substituting Eq. (13) in Eq. (14) and then integrate, we get

$q=\int_{0}^{h} 2 r\left\{\mathrm{~A} \mathrm{I}_{0}(\bar{\alpha} \mathrm{r})+\left(\frac{r^{2}}{4}+\frac{1}{\bar{\alpha}^{2}}\right) \frac{d p}{d z}-\mathrm{d} \bar{\alpha}^{2}\right\} d r$

$q=\frac{2 A h}{\bar{\alpha}} I_{1}(\bar{\alpha} h)-\mathrm{h}^{2} \mathrm{~A} I_{0}(\bar{\alpha} h)-\frac{h^{4}}{8} \frac{d p}{d z}$

From Eq. (15) we get $\frac{d p}{d z}$ as

$\frac{d p}{d z}=\frac{8 q}{h^{4} T}$

Where $T=\frac{4(\eta-1)}{k h^{3}}\left[\frac{2 h}{\bar{\alpha}} I_{1}(\bar{\alpha} h)-h^{2} I_{0}(\bar{\alpha} h)\right]-1$

$K=h \bar{\alpha}^{2} I_{0}(\bar{\alpha} h)-\bar{\alpha} I_{1}(\bar{\alpha} h)-\eta \bar{\alpha} I_{1}(\bar{\alpha} h)$

The pressure drop per wave length is given as

$\Delta p=p(0)-p(\lambda)$ is

$\Delta p=-\int_{0}^{1} \frac{d p}{d z} d z=-\int_{0}^{1} \frac{8 q}{h^{4} T} d z$

The resistance to the flow $\lambda$ is defined as

$\lambda=\frac{\Delta p}{q}=-\frac{1}{q} \int_{0}^{1} \frac{8 q}{h^{4} T} d z$

The pressure drop in the absence of stenosis $h=1$ is denoted by $\Delta p_{n}$ and is obtained from Eq. (17) as

$\Delta p_{n}=-\int_{0}^{1} \frac{8 q}{T} d z$

The resistance to the flow in the normal artery is denoted by $\lambda_{n}$ which is obtained from Eq. (19) as

$\lambda_{n}=\frac{\Delta p_{n}}{q}=-\frac{1}{q} \int_{0}^{1} \frac{8 q}{T} d z$

The normalized resistance to the flow denoted by

$\bar{\lambda}=\frac{\lambda}{\lambda_{n}}$

The shear stress acting on the wall of tube is given by

$\tau_{w}=\left(-\mu \frac{\partial u}{\partial r}\right)$ at $r=h(z)$

Introducing the dimensionless quantity

$\tau_{w}^{*}=\left(\frac{\tau_{w}}{\frac{\mu U}{d_{0}}}\right)$ at $r=h(z)$

And using Eq. (13) in Eq. (22) we get (after dropping asterisks)

$\tau_{w}=-\mu\left[A \bar{\alpha} I_{1}(\bar{\alpha} h)+\left(\frac{h}{2}\right) \frac{8 q}{h^{4} T}\right]$

The shear stress at the wall in the absence of stenosis $h=1$ denoted by $\left(\tau_{w}\right)_{n}$ is obtained from Eq. (24) as $\left(\tau_{w}\right)_{n}=-\mu\left[A \bar{\alpha} I_{1}(\bar{\alpha})+\left(\frac{1}{2}\right) \frac{8 q}{T}\right]$

The normalized shear stress at wall $\bar{\tau}_{w}$ is given by

$\bar{\tau}_{w}=\frac{\tau_{w}}{\left(\tau_{w}\right)_{n}}$

Investigation of blood flow through an artery in the presence of overlapping stenosis 


\section{Results and Discussion}

The effects of various parameters on Pressure drop $(\Delta p)$, resistance to the flow $(\bar{\lambda})$ and wall Shear stress $\left(\bar{\tau}_{w}\right)$ are given by Eq. (17), Eq. (21) and Eq. (26) respectively and have been computed numerically with the help of MATHEMATICA 9.0 Software for different values of corresponding parameters and presented graphically in Figs. 2-10.

It can be observed from the Figs.2-4 that the resistance to the flow increases with height of the stenosis for fixed values of $L_{0}, L, d, \eta, P, r, q$ and $\bar{\alpha}$. Further the resistance to the flow increases with length of stenosis (Fig. 2).These results are agree with young (1968), Gurju and Radhakrishnamacharya (2013).

It is also observed from the Figs. 3-4 that, the resistance to the flow decreases with couple stress fluid parameters $\bar{\alpha}$ and $\eta$ but this increase is insignificant for lower values of height of the stenosis $\delta \leq 0.015$.

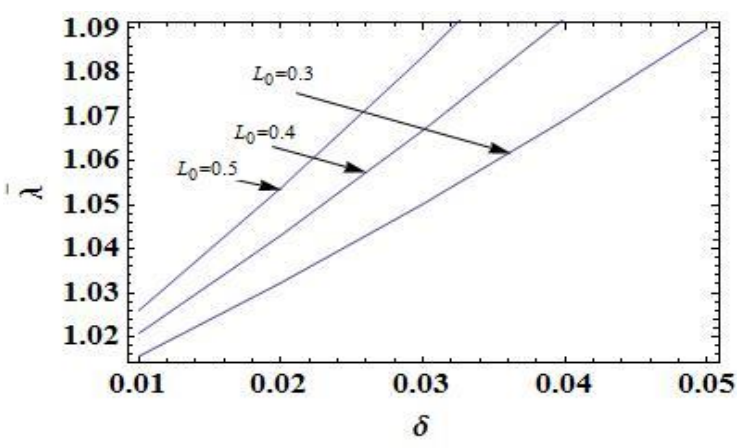

Fig. 2: Variation of flow resistance $\bar{\lambda}$ with $\delta$ for different $L_{0}(d=0.2, \bar{\alpha}=1, \eta=0.5, P=$ $0.2, r=1, L=1, q=0.2$ )

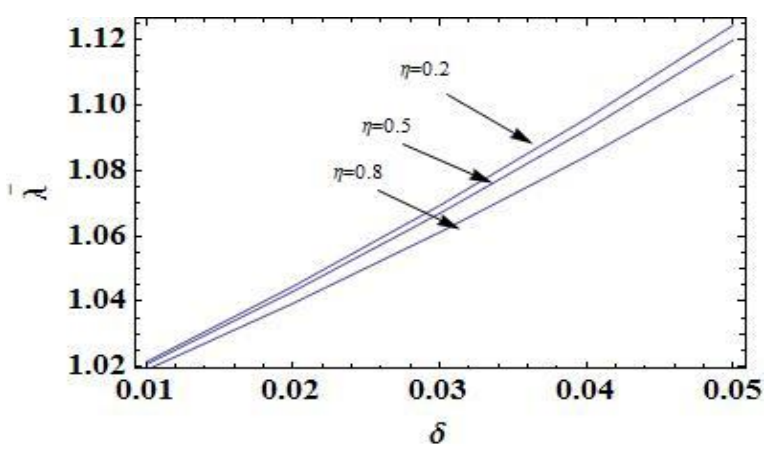

Fig. 4: Variation of flow resistance $\bar{\lambda}$ with $\delta$ for different $\eta\left(L_{0}=0.2, L=1, d=0.2, \bar{\alpha}=\right.$ $1, P=0.2, r=1, q=0.2$ )

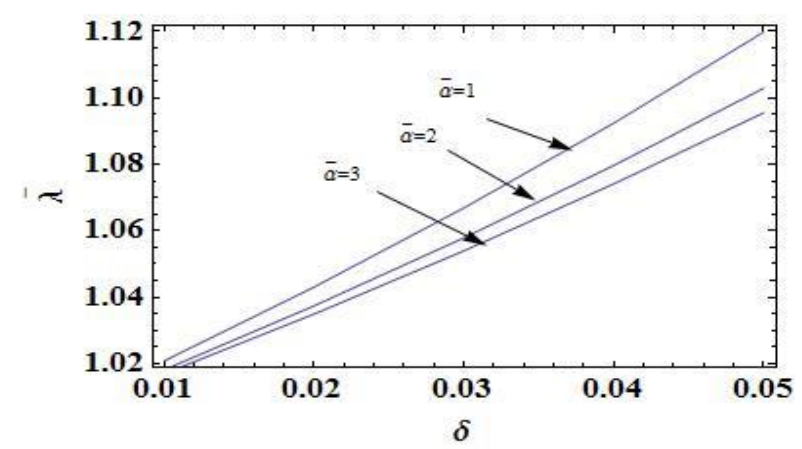

Fig. 3: Variation of flow resistance $\bar{\lambda}$ with $\delta$ for different $\bar{\alpha}\left(L_{0}=0.2, L=1, d=0.2, \eta=0.5, P=\right.$ $0.2, r=1, q=0.2$ )

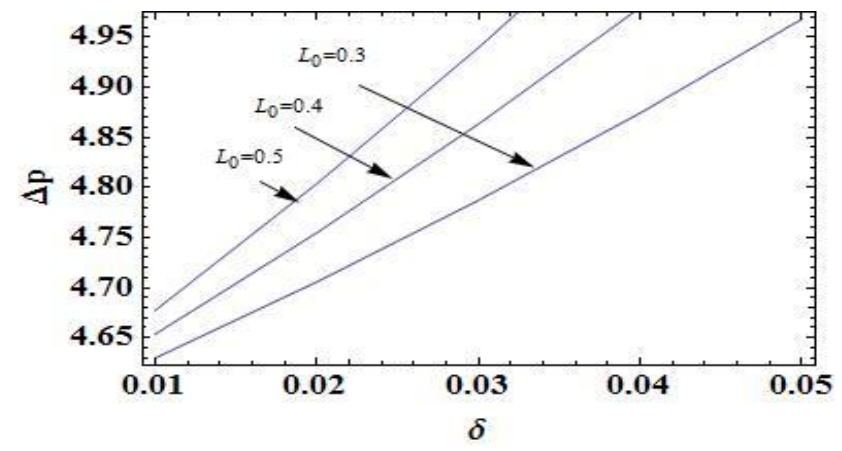

Fig. 5: Variation of pressure drop $\Delta p$ with $\delta$ for different $L_{0}(L=1, d=0.2, \bar{\alpha}=1, P=0.2, r=1, \eta=$ $0.5, q=0.2)$

It can be seen from Figs. 5 to 8 that, the pressure drop increases with height and length of the stenosis (Fig. 5). It is observed from Fig. 6 that the pressure drop increases with height of the stenosis and volumetric flow rate $(q)$. From Figs. $7 \& 8$, it can be seen that the pressure drop decreases with couple stress fluid parameters $(\bar{\alpha}, \eta)$.

Figs. 9-11 show that the wall shear stress increases with height, length of the stenosis and couple stress fluid parameters $(\bar{\alpha}, \eta)$. These results are agreed by previous results obtained by Young (1968), Gurju and Radhakrishnamacharya (2013). 


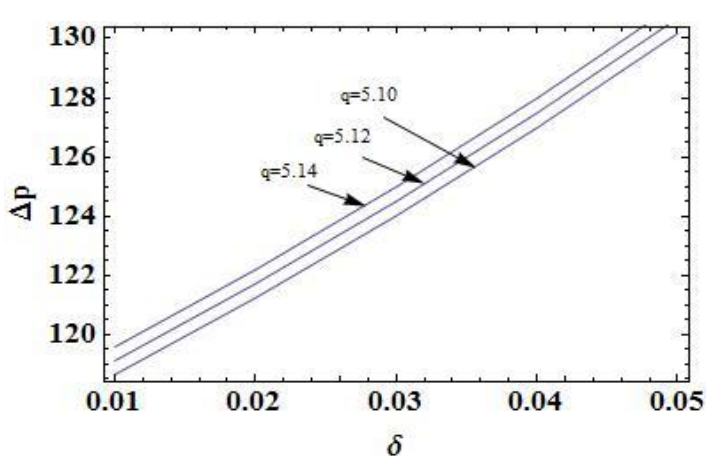

Fig. 6: Variation of pressure drop $\Delta p$ with $\delta$ for different $q\left(L_{0}=0.2, L=1, d=0.2, \bar{\alpha}=\right.$ $1, P=0.2, r=1, \eta=0.5)$

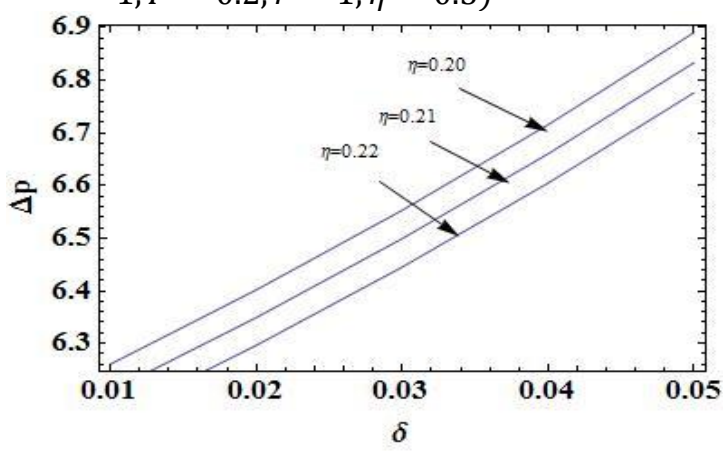

Fig. 8: Variation of pressure drop $\Delta p$ with $\delta$ for different $\eta\left(L_{0}=0.2, L=1, d=0.2, \bar{\alpha}=\right.$ $1, P=0.2, r=1, q=0.2$ )

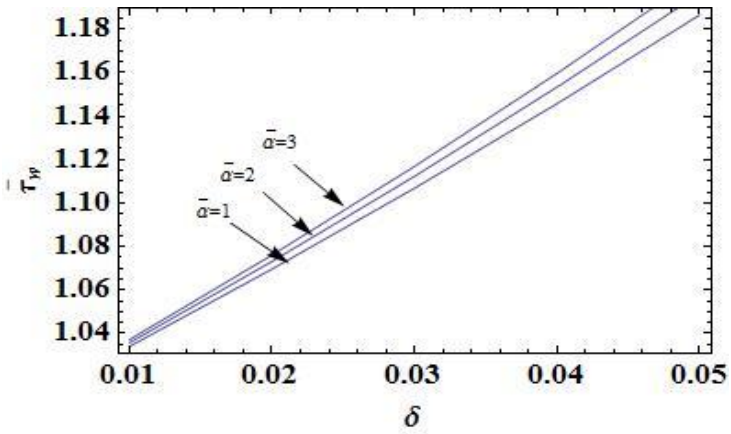

Fig. 10: Variation of shear stress $\bar{\tau}_{w}$ with $\delta$ for different $\quad \bar{\alpha} \quad(q=0.1, d=0.2, r=$ $\left.1, L_{0}=0.2, \eta=5\right)$

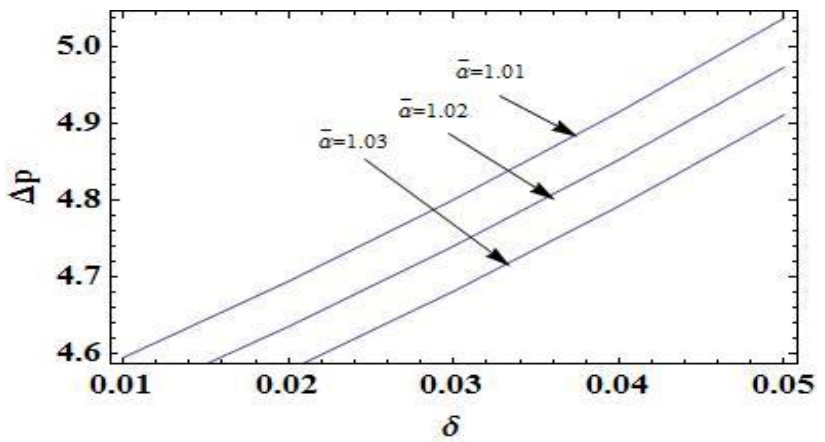

Fig. 7: Variation of pressure drop $\Delta p$ with $\delta$ for different $\bar{\alpha}\left(L_{0}=0.2, L=1, q=0.2, d=0.2, P=0.2, r=\right.$ $1, \eta=0.5)$

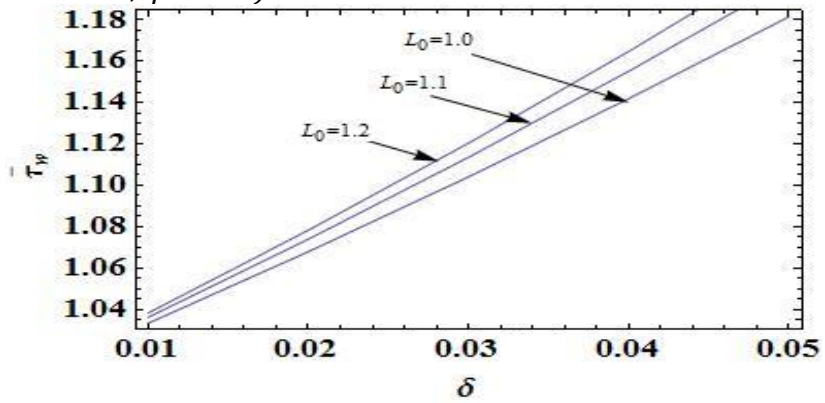

Fig. 9: Variation of shear stress $\bar{\tau}_{w}$ with $\delta$ for different $L_{0}$ $(q=0.1, d=0.2, r=1, \eta=0.5, \bar{\alpha}=1)$

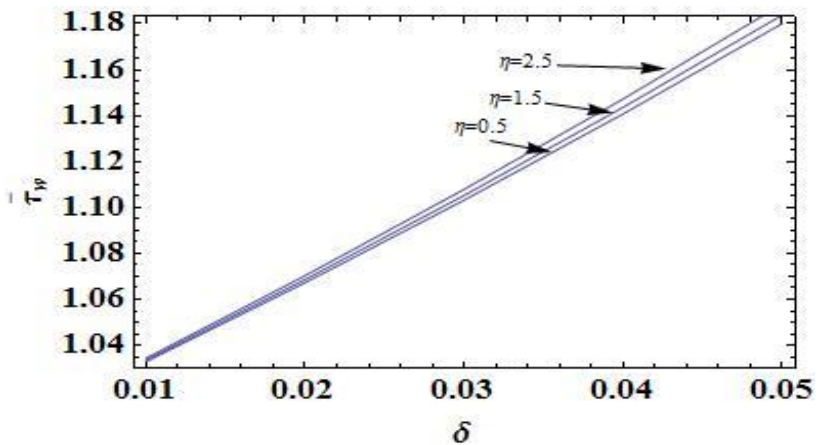

Fig. 11: Variation of shear stress $\bar{\tau}_{w}$ with $\delta$ for different $\eta$ $\left(q=0.1, d=0.2, r=1, L_{0}=0.2, \bar{\alpha}=5\right)$

\section{Conclusion}

A mathematical model for the steady flow of couple stress fluid through a tube having overlapping stenosis has been presented. Analytical solution expressions are derived in the present analysis for velocity, resistance to the flow, pressure drop and wall shear stress distribution at the stenotic region have been numerically computed for different parameters. It has been shown that the resistance to the flow increases with height of the stenosis, length of the stenosis but decreases with couple stress fluid parameters. It is also observed that the wall shear stress increases with the height of the stenosis and the couple stress fluid parameters. 


\section{References}

Gurju, A.W. and Radhakrishnamacharya, G. (2013): Effect of slip condition on couple stress fluid flow through porous medium with stenosis, International Journal of Scientific and Engineering Research, Vol. 4, Issue 9, ISSN 2229- 5518.

Arunkumar, M. (2015): Casson flow of blood through an arterial tube with overlapping stenosis, IOSR Journal of Mathematics, Vol. 11, Issue 6, pp.26-31. https://doi.org/ 10.9790/5728-11632631.

Chaturani, P. and Samy, R.P (1986): Pulsatile flow of casson's fluid through stenosed arteries with applications to blood flow, Biorheology, Vol. 23, No. 5, 499-511.

Chakravarthy, S. and Mandal, P.K. (1994): Mathematical modeling of blood flow through an overlapping arterial stenosis, Mathematical and Computer Modelling, Vol. 19, No. 1, pp. 59-70. https://doi.org/10.1016/0895-7177(94)90116-3

Fry, D. L. (1972): Localizing Factor in atherosclerosis, Atherosclerosis and Coronary Heart Disease, New York, Grune Stratton, Vol. 85.

Hamzah, B.H. and Azrar, L. (2013): Steady flow of couple-stress fluid in constricted tapered artery effects of transverse magnetic field moving catheter and slip velocity, Journal of Applied Mathematics, Vol. 2016, Article ID 9289684, 11 pages. http://dx.doi.org/10.1155/2016/9289684

Lee, J. S. and Fung, Y. C. (1970): Flow in locally - constricted tubes and low Reynolds number, Journal of Applied Mechanics, Transactions ASME, Vol. 37, pp.9-16.

Prasad, K. M. and Vijaya, R. B. and Umadevi, C. (2014): A mathematical model of Herschel- Bulkley fluid through an overlapping stenosis, IOSR Journal of Mathematics, Vol. 10, issue 2, pp.41-46. https://doi.org/10.9790/5728-10224146

Nanda, S. P. and Malik, B. (2012): A non- Newtonian two- phase fluid model for blood flow through arteries under stenotic condition, International Journal of Pharma and Bio Sciences, Vol. 2. Issue 2, pp.237-247.

Radhakrishnamacharya, G. and Srinivasarao, P. (2007): Flow of a magnetic fluid through a non- uniform wavy tube, Proceedings of the National Academy of Sciences INDIA,Vol. 76, 241-245.

Raju, M. C. and Varma, S. V. K. (2014): Soret effects due to natural convection in a non-Newtonian fluid flow in porous medium with heat and mass transfer, Journal of Naval Architecture and Marine Engineering, Vol. 11, pp. 147-156. https://doi.org/10.3329/jname.v11i2.17563

Kumar, R. S. (2015): The effect of the couple stress fluid flow on MHD peristaltic motion with uniform porous medium in the presence of slip effect, Jordan Journal of Mechanical \& Industrial Engineering ,Vol. 9, No. 4, ISSN 1995-6665 Pages 269 - 278. https://www.researchgate.net/publication/284633630

Stokes, V. K. (1966): Couple stresses in fluids, The Physics of Fluids, Vol. 9, No. 9, 1709-1715. https://doi.org/10.1063/1.1761925

Sapna, S. (2009): Analysis of non- Newtonian fluid flow in a stenosed artery, International Journal of Physical Sciences, Vol. 4 (11), pp. 663-671.

Sahu, M. K. and Sharma, S. K. and Agrawal, A. K. (2010): Study of arterial blood flow in stenosed vessel using non-Newtonian couple stress fluid model, International Journal of Dynamics of Fluids, Vol. 6, pp. $248-257$.

Srivastava,V. P. and Shailesh, S. M. (2010): Non-Newtonian arterial blood flow through an overlapping stenosis, Applications of Applied Mathematics, ISSN: 1932-9466, Vol. 5, Issue 1, pp. 225 -238.

Srinivasacharya, D. and Srikanth, D. (2012): Steady streaming effect on the flow of a couple stress fluid through a constricted annulus, Archives of Mechanics 64, 2, pp.137-152, Warszawa.

Srinivasacharya, D. and Madhavarao, G. (2016): Pulsatile flow of couple stress fluid through a bifurcated artery, Ain Shams Engineering Journal.

Texon, M. (1957): A homodynamic concept of atherosclerosis with particular reference to coronary occlusion, AMA Arch IntMed, Vol. 99, pp.418. https://doi.org/10.1001/archinte.1957.00260030100010

Verma, S.R. (2014): Mathematical modeling of Bingham plastic model of blood flow through stenotic vessel, International Journal of Engineering Research and Applications, Vol. 4, Issue 12(Part 6), pp.11-17.

Young, D. F. (1968): Effects of a time - dependency stenosis on flow through a tube, Journal of Engineering for Industry-Transactions of the ASME Vol. 90, pp.248-254. 
Zendehbudi, G. R. and Moayeri, M. S. (1999): Comparison of physiological and simple pulsatile flows through stenosed arteries, Journal of Biomechanics, Vol. 32, pp.959-965. https://doi.org/10.1016/S0021-9290(99)000536 